\title{
An Inflatable Reservoir-based Port Site Posterior Segment Drug Delivery System for Management of Age-Related Macular Degeneration and Diabetic Macular Edema
}

\author{
Pradeep Venkatesh*, Brijesh Takkar and Anubha Rathi \\ Professor of Ophthalmology, Dr RP Centre for Ophthalmic Sciences, All India Institute of Medical Sciences, India
}

\begin{abstract}
The posterior segment of the eye is often exposed to repeated surgical procedures for drug delivery while treating chronic diseases. Drug delivery systems, especially those with refillable reservoirs, are important in the perspective of diseases where continuous therapy is advocated. Port site delivery is one such system, but on-going research mentions frequently associated complications with it, particularly due to the surgical procedure involved. Herein, we hypothesize a port site delivery system based on an inflatable reservoir. Such a system would have a very simple procedure for implantation of the reservoir as well as its refilling, thereby lowering the associated complication rate.
\end{abstract}

KEYWORDS: Age related macular degeneration; Drug delivery system for retinal diseases; Intravitreal injection; Refillable reservoirs; Encapsulated cell delivery; Iontophoresis; Nanotechnology; Intravitreal reservoir

\section{INTRODUCTION}

Disorders of the posterior segment of the eye chiefly include retinal and choroidal diseases. Medical management is the gold standard for most, particularly at primary presentation. However, drug delivery to the posterior segment is severely compromised by presence of the blood retinal barriers through the systemic route, and by the ocular barriers themselves through the topical route [1].

For these reasons, intravitreal pharmacotherapy is the mainstay for most medically managed posterior segment diseases. Most common indications for intravitreal injections include vascular diseases like age related macular degeneration (ARMD) and diabetic retinopathy [2,3]. The current agents employed for treating such diseases involves repeated monthly injections of various anti VEGF agents. The recommended dosing schedule for this drug therapy necessitates up to 8-12 injections in one eye in one year, and requirement of treatment may be multiple years or indefinite [1,2]. To circumvent the complications of repeated intravitreal injections, alternate drug delivery systems (DDS) have been suggested which minimize the need for injections, and provide sustained drug delivery $[1,4,5]$. Notable amongst these are sustained release intravitreal implants, refillable implants, injectable particulate systems, encapsulated cell delivery, and iontophoresis and nanotechnology. Alternate delivery routes like trans-scleral and suprachoroidal delivery have also been suggested. Most of these systems are undergoing evaluation currently.

One of the most anticipated systems amongst these is the port site delivery system developed for injecting Ranibizumab, currently in phase 3 trials. This system involves surgically placed devices with office re-filled reservoirs. While initial trials have shown it to be efficacious, requirement for refilling may be up to 15 months [5]. However, the device is "surgically" placed and needs an incision of $>3 \mathrm{~mm}$. The procedure being partially blind, initial rates of
Quick Response Code:

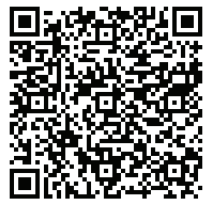

Address for correspondence: Pradeep Venkatesh, Professor of Ophthalmology, Dr RP Centre for Ophthalmic Sciences, All India Institute of Medical Sciences, India

Received: May 18, $2021 \quad$ Published: June 17, 2021

How to cite this article: Pradeep V, Brijesh T, Anubha R. An Inflatable Reservoir-based Port Site Posterior Segment Drug Delivery System for Management of Age-Related Macular Degeneration and Diabetic Macular Edema. 2021- 3(3) OAJBS.ID.000295. DOI: 10.38125/OAJBS.000295 
complications were found to be very high, as more than $50 \%$ cases developed vitreous haemorrhage. Insertion of this device requires prior surgical training to minimize complications rates [5]. A DDS with an easy and simpler implantation procedure is, therefore, highly desirable.

We hypothesize a DDS that is based on an inflatable or expandable reservoir. In contrast to other DDSs and the ones discussed before, the inflatable attribute of this DDS would make it more surgeon friendly. The large incision needed for the implant discussed before is due to the diameter of the "rigid" reservoir. Just like the usage of foldable intraocular lenses, the proposed device shall help in bringing down the incision size remarkably, while also obviating need for suturing the implant and obviating surgical training for its insertion $[1,5]$.

\section{DEVICE DESIGN}

The proposed DDS may have 3 parts: extra-scleral, scleral and intravitreal with protective septae [Figure 1]. The extra-scleral and scleral parts of the DDS may be made of non-compliant materials, whereas the intravitreal part may be made of a more compliant material that allows inflation or expansion. The extra-scleral part should be an easily identifiable solid non-transparent port that allows refilling of the intravitreal reservoir.

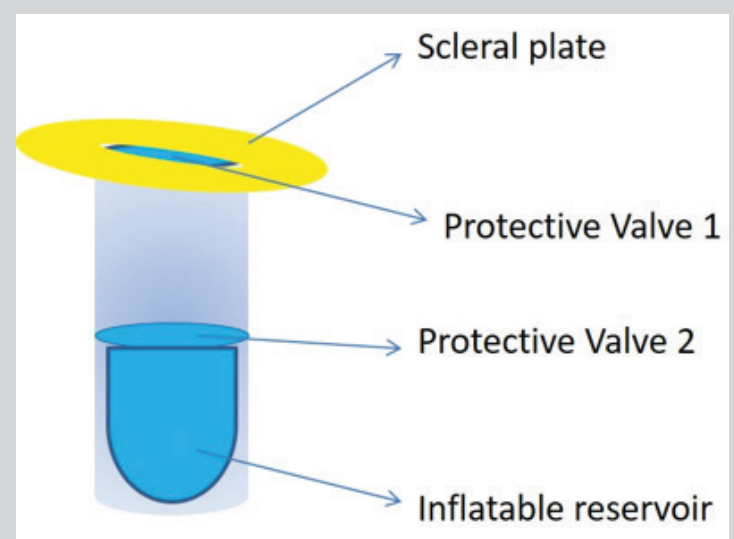

Figure 1: A schematic description of the proposed drug delivery system and its parts.

Its diameter should be slightly more than the conjoining scleral part, to prevent internal prolapse of the device. The scleral part may be around $0.5 \mathrm{~mm}$ in diameter, which is the size of a $25 \mathrm{G}$ vitreous surgery cannula [6]. Its length should be more than $0.5 \mathrm{~mm}$, so that the internal opening of the device is safely within the intravitreal cavity, and not the suprachoroidal space [7]. [The scleral and the extra-scleral parts should be separated by septum that permits passage of a refilling needle and acts as barrier. This septum has also been employed in DDSs used before [5]. The distal most "inflatable" part of the DDS should be made of a semipermeable material, which will allow continuous but controlled release of the drug.

\section{PROCEDURE}

At the outset of its implantation, the intravitreal part of the DDS can be kept folded inside the scleral conduit. Following a trans scleral stab incision with a 25 G microvitreoretinal knife in the pars plana region under aseptic precautions, the DDS can be gently inserted inside the incision manually or even with an injector [Figure 2]. The extra-scleral part of the DDS being larger in diameter than the incision, will not enter the incision site and shall be easily tucked in the sub-conjunctival space. As the length of the scleral part is more than that of the outer coat of the eye, its inner tip is now inside the intravitreal space [Figure 2].

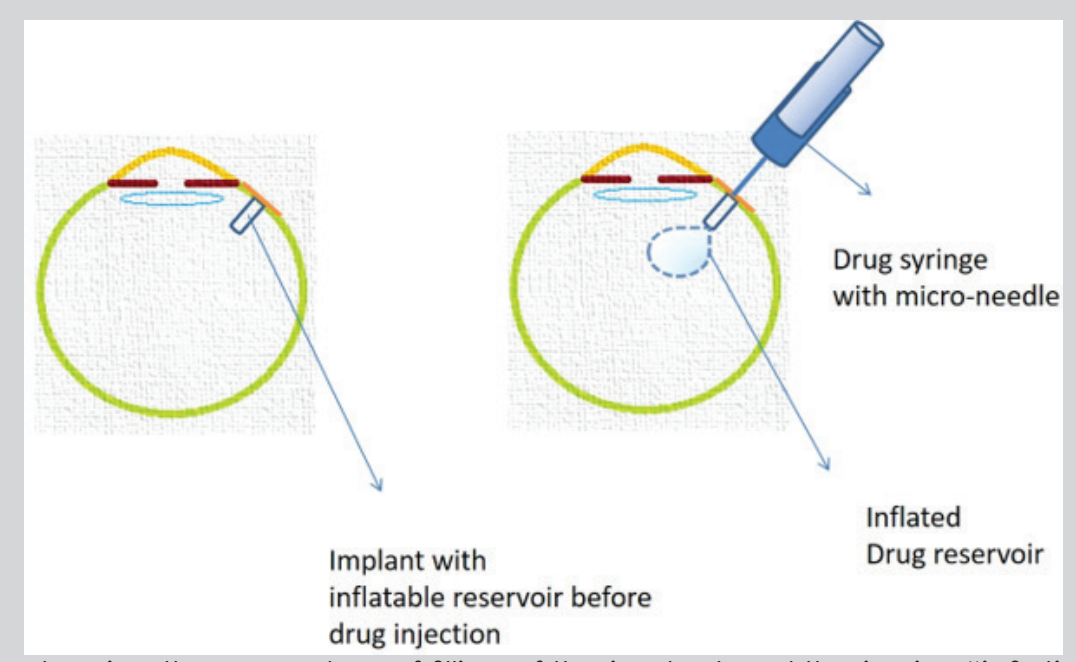

Figure 2: Illustration showing the procedure of filling of the implant and the in vivo "inflation" of the drug reservoir with the drug. 
A $27 \mathrm{G}$ needle can now be used to inject the drug in the reservoir system by penetrating the valvular septum [Figure 1,2] using a pre-filled drug injector. Prefilled drug injectors are already in use for most therapeutic agents and are easily available. Following the injection of the drug, the needle may be safely withdrawn Due to the compliant nature of the reservoir, it shall now expand to accommodate a pre-designated volume of the drug. The successful placement of the DDS can be visualized through indirect ophthalmoscopy following the procedure.

\section{REFILLING}

Following a pre-determined period or on the basis of clinical signs, the refilling of the reservoir can be planned. The refilling procedure shall involve a simple injection of the drug as in the primary procedure.

\section{REMOVAL}

If therapy is deemed completed and the drug is no longer required, the implant can be easily removed by simply hinging the external part of the DDS and gentle externalization. If required, limited conjunctival dissection may be done prior.

The current DDS have been reported to have many vision threatening complications. For example, the DDS for ranibizumab being evaluated by Genetech had very high rates of vitreous bleed, to counter which the procedure was modified. However, the modification of the procedure involves partial dissection followed by laser and cauterisation of the pars plana, and thus seems to be complicated $[1,5]$ The initial designs of the ganciclovir implant for viral retinitis by Bausch and Lomb requires a $6 \mathrm{~mm}$ incision and an anchoring suture to keep the implant in place. This implant is fraught with possibility of intraocular dislocation, particularly on its attempted removal [1]. The fluocinolone acetonide implant for posterior uveitis by Bausch and Lomb needs an incision of 3-4 mm and also an anchoring strut. This implant was reported to have very high number of complications like (migration or dislocation of the implant) in up to $90 \%$ of cases [1].

The chief difference between the DDS proposed by us and the ones existing or under evaluation is the "expandable" reservoir. This reservoir shall be advantageous in bringing down the incision size needed from $>3 \mathrm{~mm}$ to $<0.5 \mathrm{~mm}$, thus six times less. This decrease shall obviate the need for surgical training and make the procedure much easier, while retaining its efficacy. Even the refilling and the removal procedures would be simple. However, the chief advantage shall be in decreasing the complication rates related to large incision and migration of the implant.

\section{REFERENCES}

1. Bansal P, Garg S, Sharma Y, Venkatesh P (2016) Posterior segment drug delivery devices: current and novel therapies in development. Journal of Ocular Pharmacology and Therapeutics 32(3): 135-144.

2. Stewart MW (2012) Aflibercept (VEGF Trap-eye): The newest anti-VEGF drug. British Journal of Ophthalmology 96(9): 1157-1158.

3. Osaadon P, Fagan XJ, Lifshitz T, Levy J (2014) A review of anti-VEGF agents for proliferative diabetic retinopathy. Eye 28(5): 510-520.

4. Campochiaro PA, Marcus DM, Awh CC, Regillo C, Adamis AP, et al, (2019) The port delivery system with ranibizumab for neovascular age-related macular degeneration: Results from the randomized phase 2 ladder clinical trial. Ophthalmology 126(8): 1141-1154.

5. Pieramici MD. By DANTE J (2019) The port delivery system with ranibizumab retinal physician. 16: 46-49.

6. Azad SV, Takkar B. (2016) Minimally invasive vitreoretinal surgery Basics of vitrectomy In: Azad, Takkar, ed. Thieme Chapter 2. pp: 17-28.

7. Vurgese S, Panda-Jonas S, Jonas JB (2012) Scleral thickness in human eyes. PloS one 7(1): e29692. 